Journal of Luminescence 102-103 (2003) 614-617

\title{
Thermoluminescence in semiconductor-doped glasses
}

T. Miyoshi, Y. Makidera, T. Kawamura, N. Matsuo and T. Kaneda ${ }^{a}$

Department of Electrical and Electronic Engineering, Yamaguchi University, Tokiwadai, Ube, Yamaguchi 755-8611, Japan

${ }^{a}$ Ube National College of Technology, Tokiwadai, Ube, Yamaguchi 755-8555, Japan

\begin{abstract}
Thermoluminescence has been observed in X-ray irradiated CdS-doped glasses. The intensity of thermoluminescence is proportional to X-ray dose. This indicates that CdS-doped glasses are possibly used as materials for radiation dosimetry. Thermoluminescence of CdS-doped glass was compared with that of phosphor for commercial radiation dosimeter. Effects of light irradiation were also examined.
\end{abstract}

Keywords: radiation dosimeter; thermoluminescence; CdS-doped glass

Corresponding author

T. Miyoshi

Department of Electrical and Electronic Engineering, Yamaguchi University, Tokiwadai, Ube, Yamaguchi 755-8611, Japan

Fax: +81-836-85-9401

E-mail: tmiyoshi@yamaguchi-u.ac.jp 


\section{Introduction}

Thermoluminescence is observed in rare earth-doped phosphors and used for radiation dosimetry [1]. On the other hand, Grabovskis et al. [2] measured thermoluminescence in semiconductor (CdS)-doped glass, which was exposed to intense light at $80 \mathrm{~K}$, and they observed a glow peak at $120 \mathrm{~K}$. We reporeted that thermoluminescence was obsedved above room temperature in CdS-doped glasses, which were exposed to intense light at $300 \mathrm{~K}$ [3]. CdS-doped glass contains nanocrystals of CdS in glass. Thermoluminescence is explained by the following process. Electrons in the valence band of CdS nanocrystals are excited to the conduction band upon light irradiation; they migrate into the glass, and are trapped in the glass matrix. On the other hand, photogenerated holes in CdS nanocrystals migrate into the interface region between CdS nanocrystals and the glass matrix, and are trapped at defect centers. When sample is heated, trapped electrons in the glass are considered to be excited by thermal energy and recombine radiatively with holes in CdS nanocrystals as shown in Fig. 1. Thermoluminescence is also observed in X-ray irradiated CdS-doped glass [4]. Here, we report the thermoluminescence of X-ray irradiated CdS-doped glass, and it was compared with that of phosphor for commercial radiation dosimeter.

\section{Experiment}

The sample mainly investigated was commercial CdS-doped filter glass, Asahi Y-44. Absorption edge of this glass is approximately $430 \mathrm{~nm}$. The concentration of CdS was approximately $0.4 \mathrm{wt} \%$. Although thermoluminescence was observed in almost all glasses investigated, Asahi L-42, Y-44, Y-46, Y-48; Hoya L-42, Y-44, Y-46, Y-48; Schott GG420, GG435, GG455, GG475; and Corning 3-74, 3-73, 3-72 and 3-71, Asahi Y-44 showed the most intense thermoluminescence. The glass was exposed to X-ray from an X-ray diffractometer (Rigaku RV-200; $\mathrm{Cu}$ target, $40 \mathrm{kV}$ ) at $300 \mathrm{~K}$. Thermoluminescence was measured using a TLD (thermoluminescence dosimeter) reader (Kyokko 2500). The glow curve was recorded using a pen recorder. Thermoluminescence of phosphor for commercial TLD, Kyokko MSO $\left(\mathrm{Mg}_{2} \mathrm{SiO}_{4}: \mathrm{Tb}\right)$, was also measured for comparison. 


\section{Results and discussion}

Thermoluminescence measurements were performed by heating the irradiated sample to $770 \mathrm{~K}$. The heating rate was $4 \mathrm{~K} / \mathrm{s}$. The solid curve in Fig. 2 shows the glow curve of thermoluminescence for Asahi Y-44. One glow peak is observed at $480 \mathrm{~K}$. Thermoluminescence is considered to be observed by the process shown in Fig.1: the trapped electrons in glass are excited by thermal energy and recombine radiatively with holes in CdS nanocrystals. From the glow curve, we evaluate the location of traps using the initial rise method [1]. The initial rise part of a thermoluminescence curve is exponentially dependent on temperature according to $I=A \exp (-E / k T)$,

where $A$ is a constant, $E$ is the energy difference between the traps and the conduction band edge of glass, and $k$ is Boltzmann's constant. We evaluate the value of $E=1 \mathrm{eV}$ from a plot of $\ln (I)$ versus $1 / T$. Since glass is an amorphous material, the traps are thought to be distributed in energy. A broad peak and tail on the high-temperature side indicate that this assumption is valid.

The distribution of traps is confirmed by the following technique [1]. The sample is heated to a temperature $T_{\mathrm{s}}$ corresponding to a position on the low temperature tail of the glow peak. The sample is then cooled to room temperature and reheated, at the same linear rate, to $770 \mathrm{~K}$. The position of the first maximum, $T_{\mathrm{m}}$, is noted. The whole process is then repeated on a freshly irradiated sample using a new value of $T_{\mathrm{s}} \cdot T_{\mathrm{m}}-T_{\mathrm{s}}$ curve of thermoluminescence for Asahi Y-44 is a straight line with a slope of 1 [3]. This indicates a quasi-continuous distribution of peaks arising from the distribution of trap depth. The value of $E=1 \mathrm{eV}$ indicates the position of the traps nearest to the conduction band of glass. If trap level is not distributed, the value of $T_{\mathrm{m}}$ is not changed with changing $T_{\mathrm{s}}[1]$.

The broken curve in Fig. 2 shows the glow curve of thermoluminescence for MSO. Main glow peak is observed at $500 \mathrm{~K}$. Luminescence intensity for MSO is approximately 20 times of that for Y-44.

The intensity of thermoluminescence was measured as a function of X-ray dose. Fig. 3 shows the result. The intensity of thermoluminescence is proportional to X-ray dose. This indicates that CdS-doped glasses are possibly used as materials for radiation dosimetry. The intensity of thermoluminescence depends on photon energy of X ray and gamma ray. The energy response depends on materials, and it may be tailored by changing glass composition. 
Thermoluminescence is observed in light irradiated CdS-doped glasses [3,4]. Effect of light irradiation was examined using a fluorescent lamp. Fig. 4 shows glow curves of light irradiated CdS-doped glass, Y-44 (solid cueve), and MSO (dashed curve). Glow curve of thermoluminescence for light irradiated CdS-doped glass is the same as that of X-ray irradiated sample. On the contrary, glow curve for light irradiated MSO is different from that of X-ray irradiated sample. Irradiation intensity for Y-44 is 6 times of that for MSO. Luminescence intensity for Y-44 is approximately $30 \%$ of that for MSO. Therefore, luminescence intensity for Y-44 is approximately $5 \%$ of that for MSO, if irradiation intensity for Y-44 is the same as that for MSO. Although the intensity of thermoluminescence for CdS-doped glass after X-ray irradiation is weaker than that for MSO, effect of light is smaller than that for MSO.

Fig. 5 shows the intensity of thermoluminescence as a function of irradiation intensity for Y-44. Irradiation time is $2 \mathrm{~h}$. The intensity of thermoluminescence is almost proportional to irradiation intensity. Fig. 6 shows the intensity of thermoluminescence as a function of irradiation time for Y-44. Light intensity is $1500 \mathrm{~lx}$. The intensity of thermoluminescence increases linearly with increase in irradiation time.

If X-ray irradiated sample is exposed to light, the trapped electrons in glass are excited by photon energy and recombine with holes in CdS nanocrystals. Thus, thermoluminescence intensity may decrease. Y-44 and MSO were exposed to light after X-ray irradiation. Irradiation time of X-ray is $8 \mathrm{~min}$, and current of X-ray tube is $10 \mathrm{~mA}$. X-ray dose is $40 \mathrm{mGy}$. Light intensity is $9000 \mathrm{~lx}$, and irradiation time is $2 \mathrm{~h}$. The intensity of thermoluminescence after exposure to light is approximately $50 \%$ of that before exposure to light for MSO. On the other hand, the noticeable decrease in luminescence intensity was not observed for Y-44. Thermal fading for Y-44 may be as small as that for MSO, since the temperature of the glow peak is as high as that for MSO.

ESR (electron spin resonance) signal has been observed in X-ray irradiated CdS-doped glass [5]. The X-ray induced signal is proportional to X-ray dose. This indicates that an ESR spectrometer is possibly used as a dosimeter reader. ESR signal is measured nondestructively, since trapped electrons in glass stay in the traps during the measurement. On the contrary, thermoluminescence is measured destructively. However, sensitivity of thermoluminescence is higher than that of ESR. 


\section{Conclusion}

Thermoluminescence has been observed in X-ray irradiated CdS-doped glasses. The intensity of thermoluminescence is proportional to X-ray dose. This indicates that CdS-doped glasses are possibly used as materials for radiation dosimeter. The intensity of thermoluminescence for Asahi Y-44 is approximately $5 \%$ of that for phosphor for commercial TLD $\left(\mathrm{Mg}_{2} \mathrm{SiO}_{4}: \mathrm{Tb}\right)$. Although the intensity of thermoluminescence for CdS-doped glass after X-ray irradiation is weaker than that for MSO, effect of light irradiation is smaller than that for MSO. 


\section{References}

[1] S. W. S. McKeever, Thermoluminescence of solids, eds. R. W. Cahn, E. A. Davis, I. M. Ward (Cambridge University Press, Cambridge, 1985).

[2] V. Ya. Grabovskis, Ya. Ya. Dzenis, A. I. Ekimov, I. A. Kudryavtsev, M. N. Tolstoi, U. T. Rogulis, Sov. Phys. Solid State 31 (1989) 149.

[3] T. Miyoshi, H. Sera, N. Matsuo, T. Kaneda, Jpn. J. Appl. Phys. 40 (2001) 2327.

[4] T. Miyoshi, Y. Makidera, T. Kawamura, S. Kashima, N. Matsuo, T. Kaneda, Jpn. J. Appl. Phys. 41 (2002) 5262.

[5] T. Miyoshi, H. Furukawa, K. Nitta, H. Okuni, N. Matsuo, Solid State Commun. 104 (1997) 451. 


\section{Figure captions}

Fig. 1 Simple models for thermoluminescence (TL) in semiconductor-doped glass and rare earth-doped phosphor.

Fig. 2 Glow curves of thermoluminescence for CdS-doped glass, Asahi Y-44 (solid curve), and phosphor for commercial TLD, MSO $\left(\mathrm{Mg}_{2} \mathrm{SiO}_{4}\right.$ : $\left.\mathrm{Tb}\right)$ (dashed curve). Luminescence intensities at glow peakes are normalized. Luminescence intensity for Y-44 is approximately $5 \%$ of that for MSO. Current of the X-ray tube is $10 \mathrm{~mA}$, and irradiation time is $8 \mathrm{~min}$. X-ray dose is $40 \mathrm{mGy}$, which was measured by the TLD reader using MSO.

Fig. 3 Intensity of thermoluminescence as a function of X-ray dose. Solid circles represent luminescence intensity as a function of irradiation intensity (current of X-ray tube). Irradiation time is $8 \mathrm{~min}$. Open triangles represent luminescence intensity as a function of irradiation time. Current of X-ray tube is $200 \mathrm{~mA}$.

Fig. 4 Glow curves of thermoluminescence for Asahi Y-44 (solid curve) and MSO ( $\left.\mathrm{Mg}_{2} \mathrm{SiO}_{4}: \mathrm{Tb}\right)$ (dashed curve). Light intensity is $9000 \mathrm{~lx}$ for Y-44 and $1500 \mathrm{~lx}$ for MSO. Irradiation time is $2 \mathrm{~h}$.

Fig. 5 Intensity of thermoluminescence as a function of irradiation intensity for Asahi Y-44. Irradiation time is $2 \mathrm{~h}$.

Fig. 6 Intensity of thermoluminescence as a function of irradiation time for Asahi Y-44. Irradiation intensity is $1500 \mathrm{~lx}$. 
Fig. 1

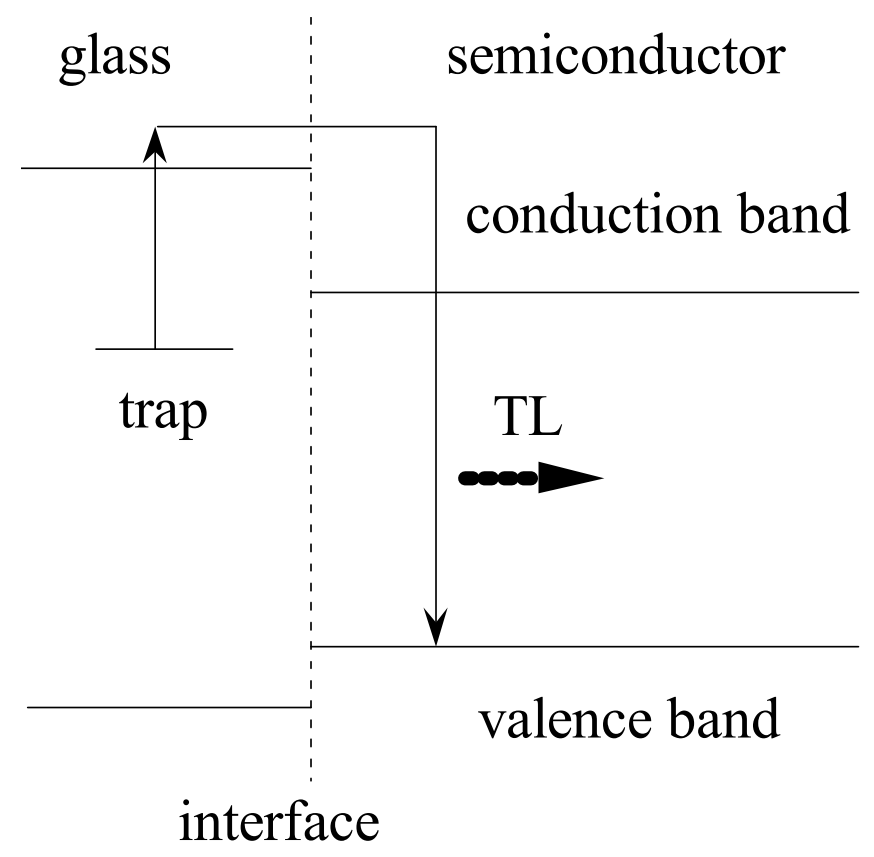

conduction band

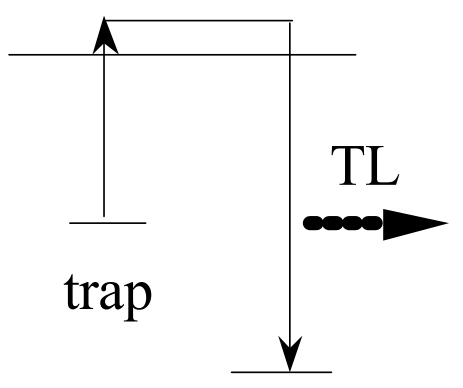

semiconductor-doped glass

phosphor 
Fig. 2

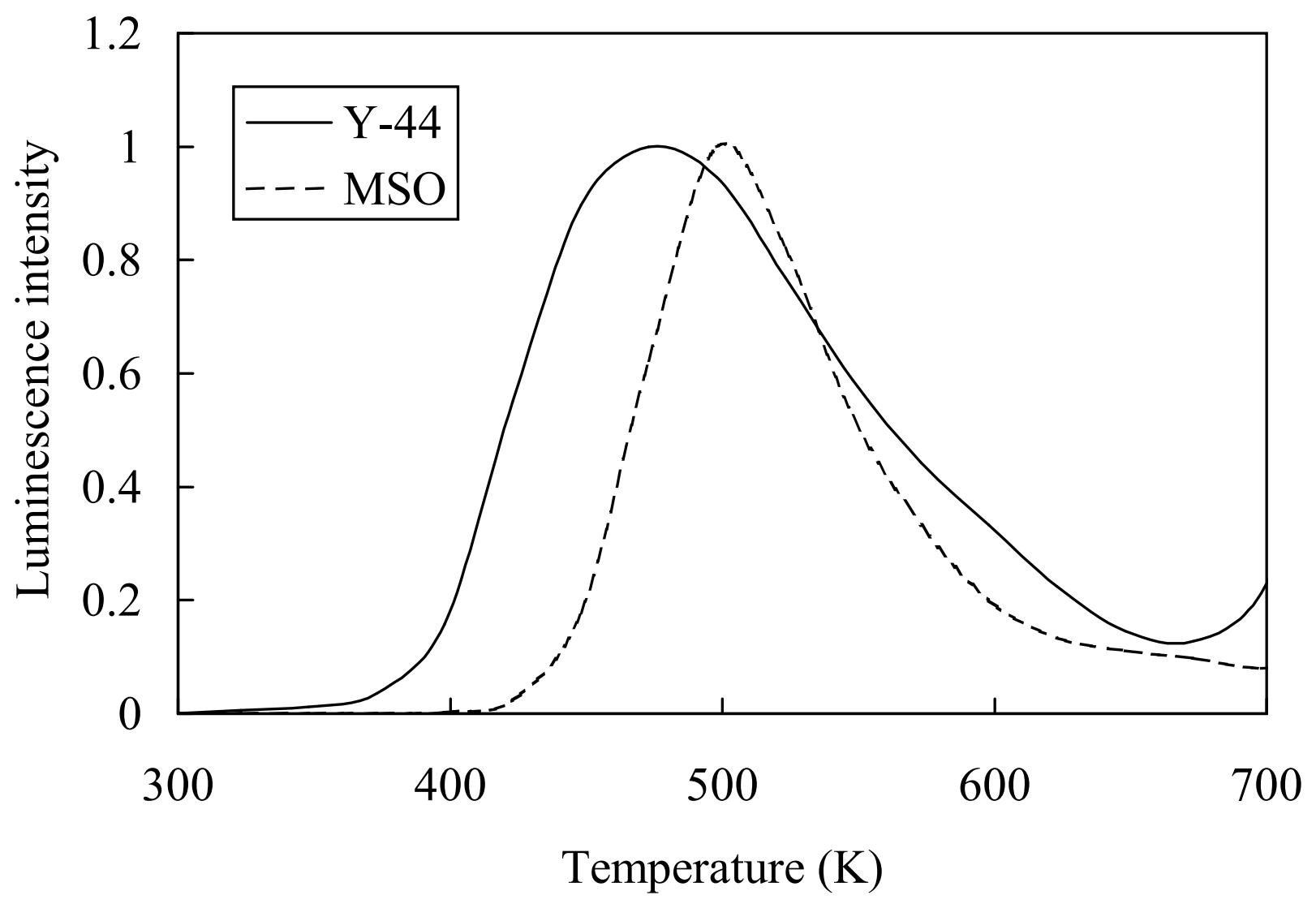


Fig. 3

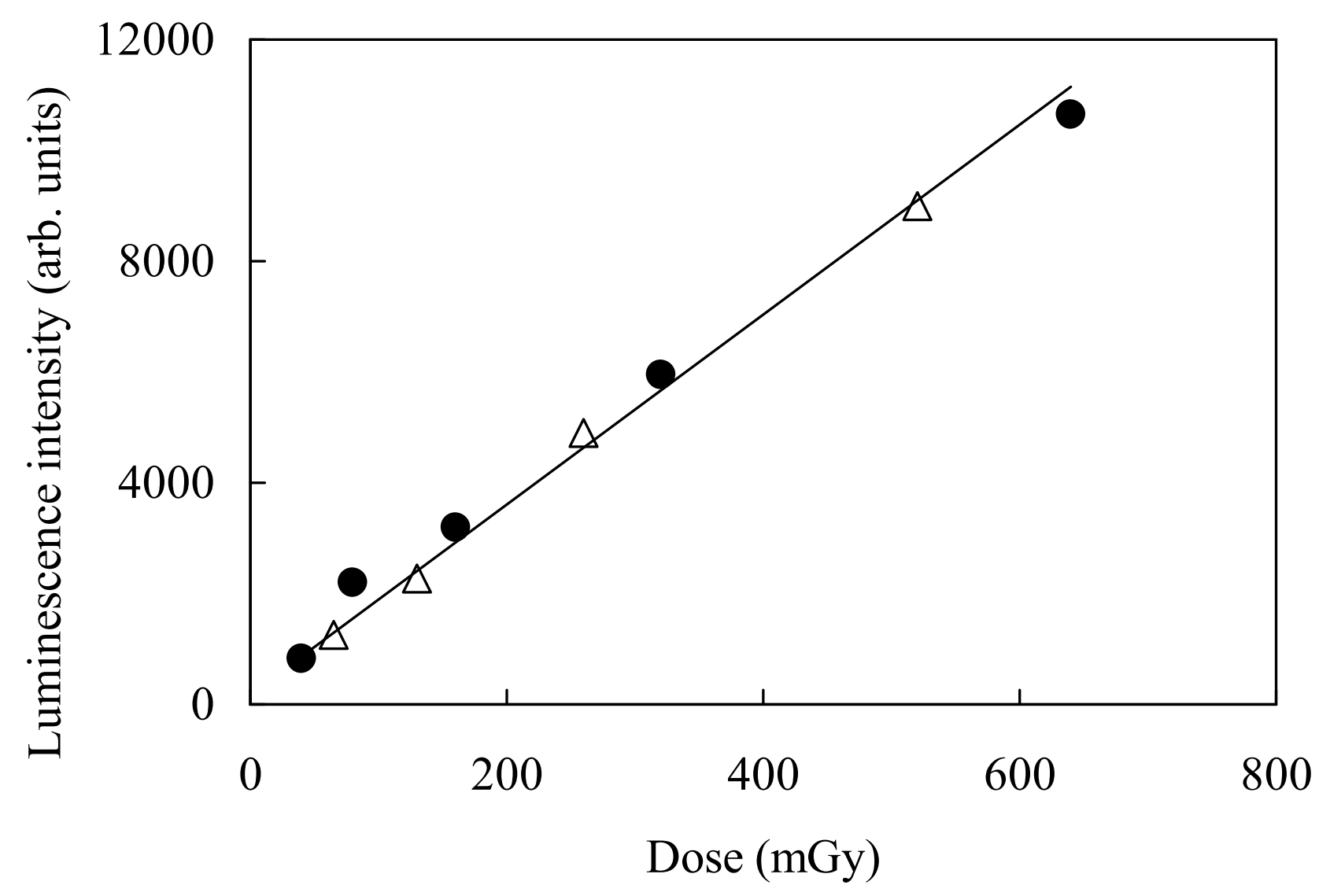


Fig. 4

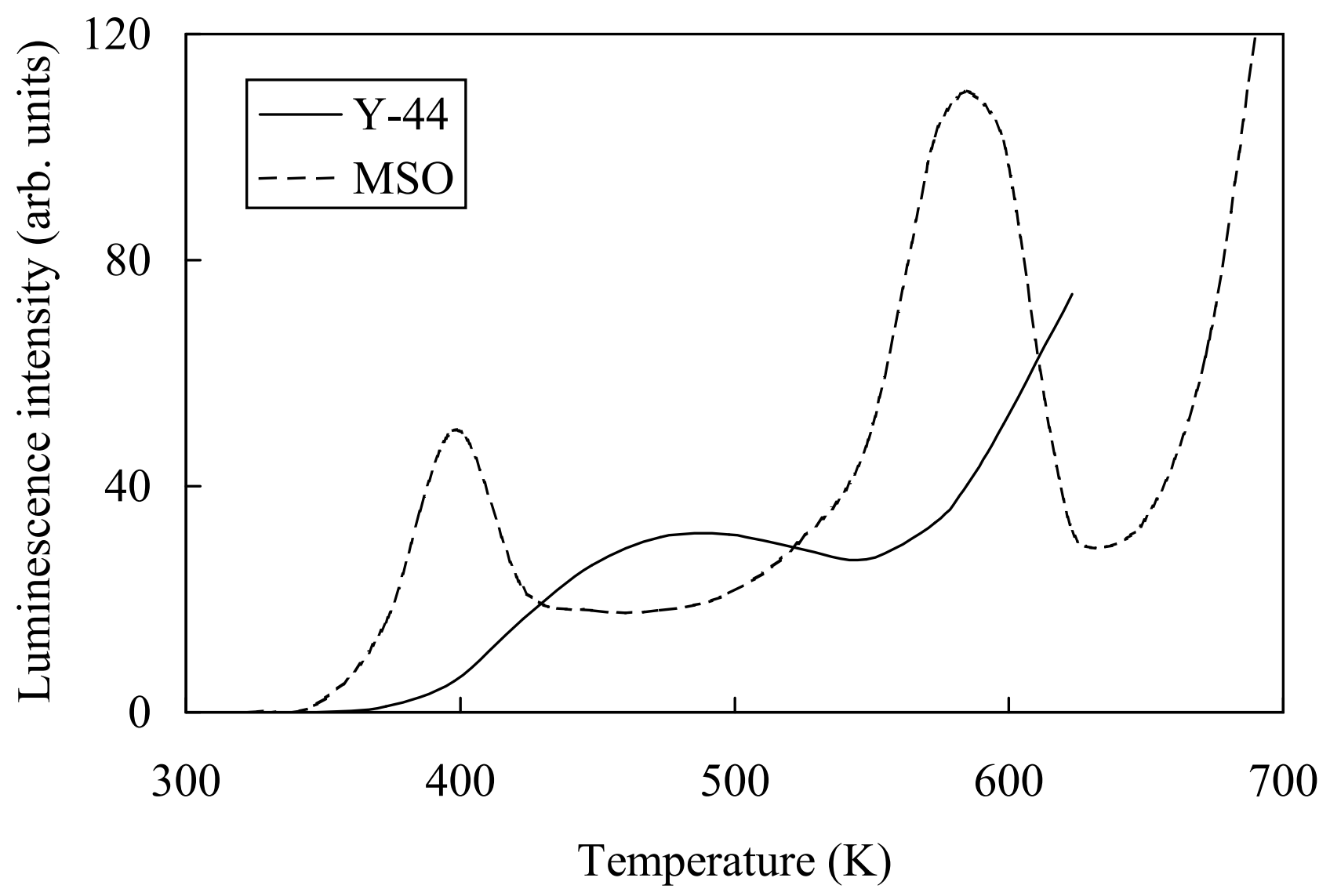


Fig. 5

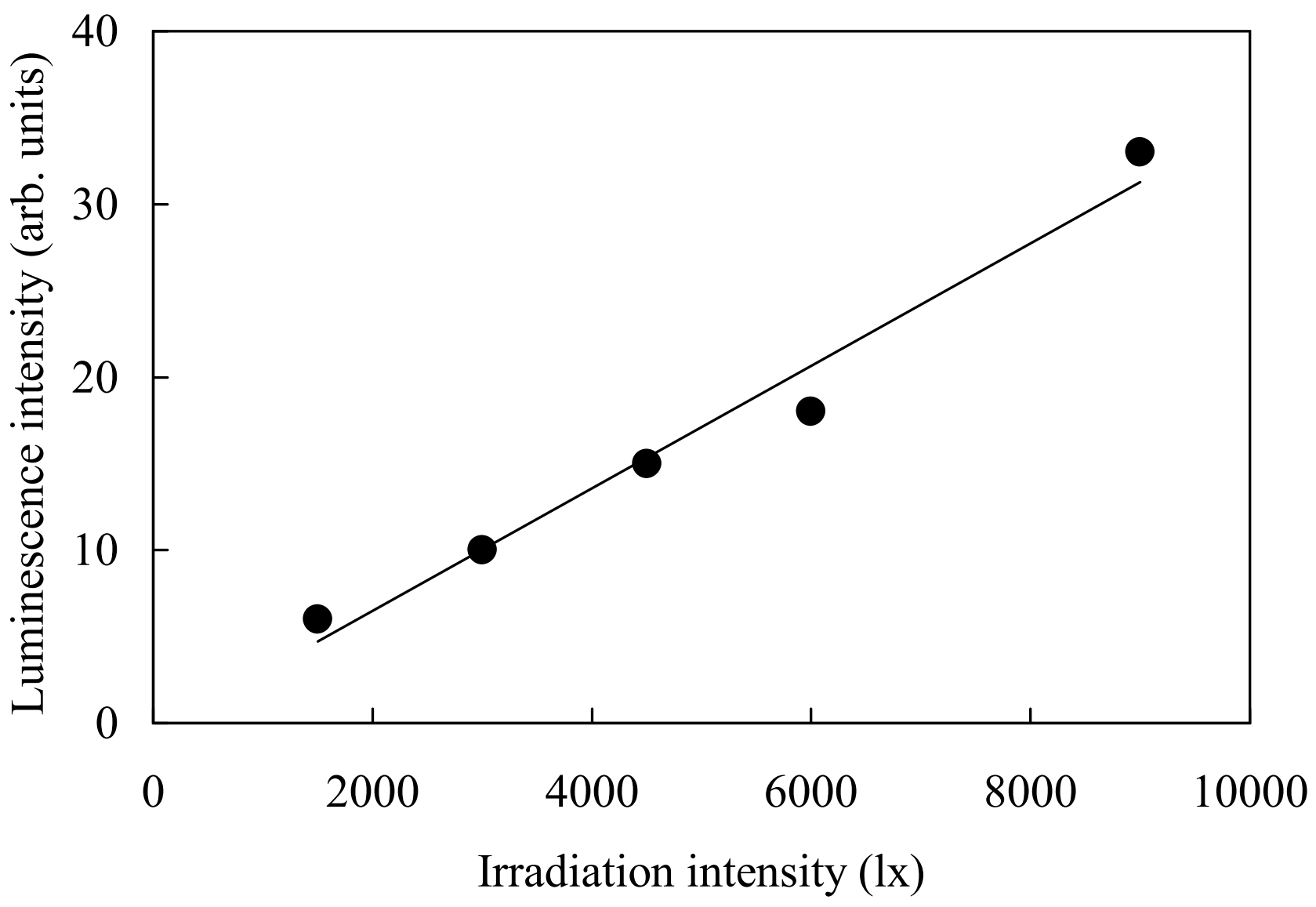


Fig. 6

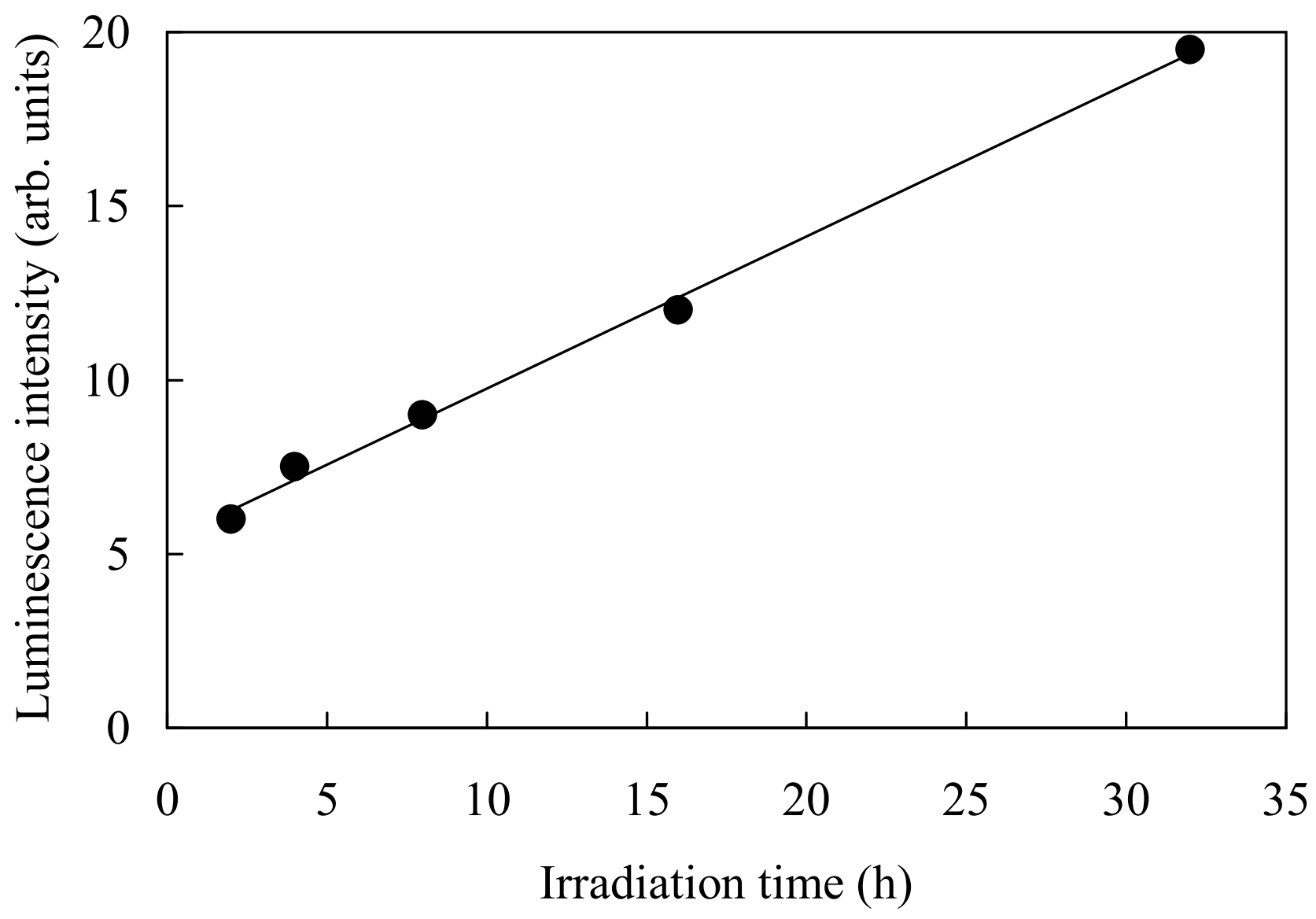

